\title{
Analysis of Work-of-Fracture Method for MEASURing Fracture Energy of Concrete
}

\author{
By Zdeněk P. Bažant, ${ }^{1}$ Fellow, ASCE
}

\begin{abstract}
The role that plastic-frictional energy dissipation in the fracture process zone plays in the workof-fracture method for measuring the fracture energy of concrete or other quasibrittle materials is analyzed, and a possible improvement of this method is proposed. It is shown that by measuring the unloading compliance at a sufficient number of states on the post-peak descending load-deflection curve, it is possible to calculate the pure fracture energy, representing the energy dissipated by the fracture process alone. However, this value of fracture energy is pertinent only if the material model (constitutive law and fracture law) used in structural analysis takes into account separately the fracture-damage deformations and the plastic-frictional deformations. Otherwise, one must use the conventional fracture energy, which includes plastic-frictional energy dissipation. Either type of fracture energy should properly be determined by extrapolation to infinite specimen size. Further, it is shown that the unloading compliancies to be used in the calculation of the pure fracture energy can be corrected to approximately eliminate the time-dependent effects (material viscoelasticity) and reverse plasticity. Finally, it is proposed to improve the work-of-fracture method by averaging the work done by fracture over only a central portion of the ligament. However, experiments are needed to check whether the specimen size required for this improved method would not be impracticably large.
\end{abstract}

\section{INTRODUCTION}

The work-of-fracture method, which was proposed for ceramics by Nakayama (1965) and Tattersal and Tappin (1966), and for concrete by Hillerborg et al. (1976) [also Hillerborg (1983, 1985) and RILEM (1985)], is one effective method for measuring the fracture energy $G_{f}$ of concrete. This method, which is attractive by its simplicity of concept, is based on the cohesive (or fictitious) crack model, which was proposed in a simplified form by Barenblatt $(1959,1962)$ and was developed in detail and applied to concrete by Hillerborg and co-workers (Hillerborg et al. 1976; Petersson 1981; Hillerborg 1983, 1985).

Since the cohesive crack model is a fracture model, questions regarding the validity and meaning of this approach arise with respect to the plastic-frictional dissipation of energy. Other questions arise with respect to the variability of the energy (per unit crack area) required for breaking the material. The purpose of this paper is to analyze these questions and clarity the meaning of fracture energy obtained by the workof-fracture method.

\section{COHESIVE CRACK MODEL AND ITS BASIC HYPOTHESES}

The usual and simplest version of the cohesive (or fictitious) crack model rests on two basic hypotheses (normally only tacitly implied):

1. There exists a unique function $\varphi$ such that

$$
\sigma=\varphi(v)
$$

where $\sigma=$ cohesive (crack-bridging) stress; $v=$ upper surface displacement (half-crack opening) (Fig. 1). This means that function $\varphi$ is assumed independent of position $x$ and of the structure size characterized by dimension $D$.

2. There is no energy dissipation outside the cohesive crack.

\footnotetext{
${ }^{1}$ Walter P. Murphy Prof. of Civ. Engrg. and Mat. Sci., Northwestern Univ., Evanston, IL 60208.

Note. Associate Editor: Robert Y. Liang. Discussion open until July 1, 1996. To extend the closing date one month, a written request must be filed with the ASCE Manager of Journals. The manuscript for this paper was submitted for review and possible publication on November 14, 1994. This paper is part of the Journal of Engineering Mechanics, Vol. 122, No. 2, February, 1996. CASCE, ISSN 0733-9399/96/0002$0138-0144 / \$ 4.00+\$ .50$ per page. Paper No. 9580 .
}

Further, it is assumed that fracture can be treated as a timeindependent process, although in reality it is not.

For determining the length of the cohesive crack, of course, it is also required that the total stress-intensity factor caused (at the cohesive crack tip) by both the applied load $P$ and the crack-bridging stresses $\sigma(x)$ be zero. Without this condition, the length of the cohesive crack would be indeterminate and the complementary energy would not be minimized with respect to the crack length (Bažant and $\mathrm{Li} 1995$ ). This condition, however, does not enter the analysis that follows.

The fracture energy $G_{f}$ of the material is, in the classical cohesive crack model, defined as

$$
G_{f}=2 \int_{v=0}^{v+\infty} \varphi(v) d v
$$

which is the work done by $\sigma$ during the breaking of the material per unit crack area. According to hypothesis 2, the energy dissipated by complete fracture of the specimen is $b(D$ $\left.-a_{0}\right) G_{f}$ where $D=$ cross-section dimension; $a_{0}=$ length of the notch or initial traction-free crack; $D-a_{0}=$ length of the ligament; and $b=$ specimen thickness (Fig. 1).

On the other hand, regardless of the actual material behavior, the energy required to completely break the specimen is the work done by load $P$ on the load-point deflection $w$, which is equal to area $A$ under the complete load-deflection curve $P(w)$. Accordingly, under the assumption that all work of the load is dissipated by the cohesive crack, the work-of-fracture method determines the apparent fracture energy as

$$
G_{f a}=\frac{A}{b\left(D-a_{0}\right)}=\frac{1}{b\left(D-a_{0}\right)} \int_{0}^{\infty} P(w) d w
$$

If the material does indeed follow the cohesive crack model, then

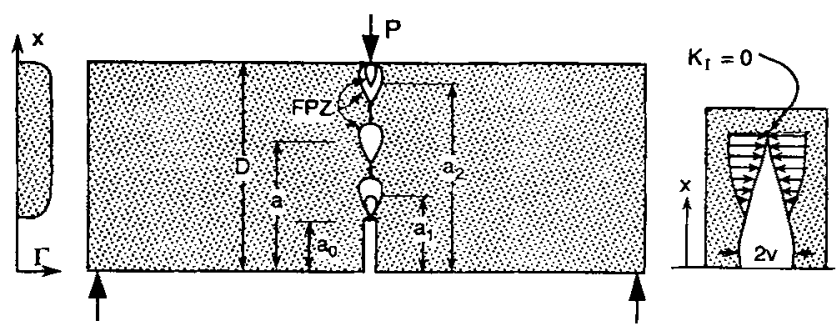

FIG. 1. Typical Test Specimen and Cohesive Crack 


$$
G_{f}=G_{f a}
$$

Eq. (3) with (4) represents the basis of the work-of-fracture method, which is one of three RILEM (1985) Standard Recommendations for the measurement of fracture energy of concrete (the other two are the two-parameter method of Jenq and Shah, and the size-effect method of Bažant). It is clear that the work-of-fracture method relies on the foregoing two hypotheses. These, however, are never fulfilled exactly and probably involve a significant error, which has two sources.

One source of the probable error is that a significant part of the energy may be dissipated by plasticity with friction, both within the specimen and at the supports (Planas and Elices 1989), thus violating hypothesis 2 . The energy dissipated by irreversible processes far from the crack (typically by friction and material crushing at the supports) can be adequately handled by proper design of the experimental setup (Planas and Elices 1989; Guinea et al. 1992).

The second source of error is that the softening law $\varphi(v)$ can be nonunique. One reason is that the actual fracture process zone, which consists of a zone of microcracking ahead of the crack tip, may vary its width during propagation, and the distribution of microcracking intensity across the process zone may also vary. The nonuniqueness of $\varphi(v)$ may arise because $v$ represents the sum of the widths of all the microcracks in a cross section of the fracture process zone. Furthermore, unless the fracture process zone is far smaller than the cross-section dimension, the effective width of this zone depends on the specimen size, which causes a size effect. These phenomena are clearly seen in fracture simulations with random particle systems (or discrete-element method), such as those described in Zubelewicz and Bažant (1987), Bažant et al. (1990), and Jirásek and Bažant (1994, 1995), and are indicated by some experimental observations [e.g., Mihashi et al. (1991)]. There are other reasons for the nonuniqueness of $\varphi(v)$. Similar to the more sophisticated nonlocal or gradient material models, the curve $\varphi(v)$, too, must be affected by triaxial material behavior in the fracture process zone. In particular, it must depend on the normal stresses or strains parallel to the crack plane, which vary during the test and are different for different specimen geometries and sizes.

The energy dissipated by complete fracture can also be expressed as

$$
W_{f}=\int_{x=a_{0}}^{x=D} \Gamma(x) d x, \text { with } \Gamma(x)=2 \int_{u=0}^{u=\infty} \sigma(x) d v(x)
$$

where $x=$ coordinate measured from the mouth of crack or notch; and $\Gamma=$ breaking energy = energy dissipated by a total break of the material per unit area of one surface of the main crack. Note that we avoid calling $\Gamma$ the surface energy, because this term is used in physics and thermodynamics for the Gibbs free energy of the solid surface, which corresponds to the energy required to create one smooth crack surface. In quasibrittle materials, much more energy is dissipated by microcracks and frictional slips on the sides. This energy, which is much larger than the surface Gibbs free energy, should be counted into $\Gamma$, as must the excess surface energy due to the microscopic tortuosity of the final fracture.

According to the assumptions of the cohesive crack model, $\Gamma(x)$ is a constant. This is not true in reality, which follows, for example, from the analysis of $\mathrm{Hu}$ and Wittmann (1991, 1992). Consequently, the apparent fracture energy should be understood as the mean value of $\Gamma$ over the ligament

$$
G_{f a}=\frac{1}{b\left(D-a_{0}\right)} \int_{a_{0}}^{D} \Gamma(x) d x
$$

Because, in general, $\Gamma$ is not a material property, neither is
$G_{f a}$. Thus, as is well-known, $G_{f a}$ depends on the geometry and size of specimen. However, the limit for infinite size $(D \rightarrow \infty)$ does provide a value that is a material property. The reason (Bažant 1987a, b; Bažant and Pfeiffer 1987) is that in an infinitely large specimen, the fracture process zone boundary is exposed to the same asymptotic near-tip displacement field of linear elastic fracture mechanics (LEFM), regardless of the specimen geometry. This is true for a crack tip at any point of the ligament. So, $\Gamma$ is independent of $x$ in a specimen of infinite size. Thus a rigorous definition of fracture energy representing a material constant can be stated as

$$
G_{f}=\lim _{D \rightarrow \infty} G_{f a}=\lim _{D \rightarrow \infty} \Gamma
$$

(Note, therefore, that if the specimen size is extrapolated to infinity, LEFM suffices for defining $G_{f}$. The size-effect method of Bažant directly exploits this fact.)

It is usually thought that, in the sense of the cohesive crack model, $G_{f}$ represents (or at least ought to represent) exclusively the energy dissipated by the physical processes of fracturing. This is not so, however. This important question will be analyzed next.

\section{ROLE OF PLASTIC-FRICTIONAL ENERGY DISSIPATION IN FRACTURE}

Consider a fracture specimen (Fig. 1) loaded under displacement control. The testing machine continuously adjusts the applied load $P$ as a function of the load-point displacement $w$ so as to keep equilibrium, and so $P$ is defined as a function of $w, P=P(w)$. The potential energy $\Pi$ of the structure-load system is the energy that can be recovered from the system by unloading. The incremental work of the load $P$ applied by the testing machine is $P d w$ and the potential energy increment of the load $-P d w$. According to the principle of conservation of energy, the potential energy increment $d \Pi$ between points 1 and 2 on the load-deflection curve (Fig. 2) represents the sum of the potential energy increments of the specimen (structure) and the load, that is

$$
d \Pi=d U-P d w
$$

in which

$$
U=w_{r}^{2} / 2 C_{u}
$$

Here $C_{u}=$ unloading compliance $=$ inverse slope of line 13 in Fig. $2 ; w_{r}=$ recoverable (or reversible) displacement $=$ horizontal projection 37 of line 31 in Fig. 2; and $U=$ strain energy of the specimen, representing the energy that can be recovered by unloading, with the graphical meaning $U=$ area of the triangle 1371 under the unloading diagram 13 in Fig. 2. Note that the potential energy of the load is not $-P w$ because the load is not a gravity load (a fracture test cannot be conducted under load control).

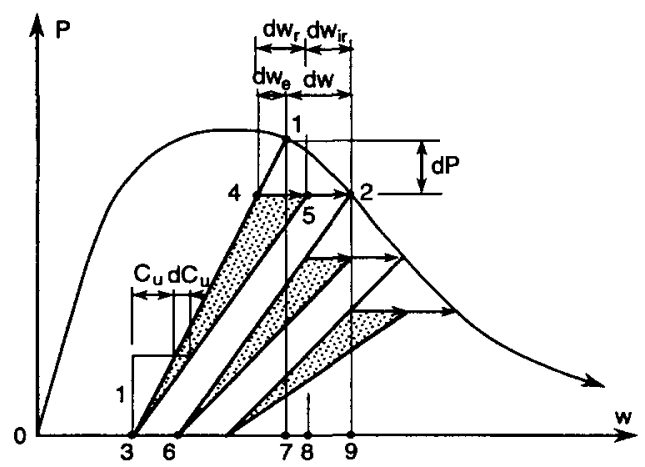

FIG. 2. Separation of Energles Dissipated by Fracture and by Plasticity with Friction 
As known from experiments [e.g., Wecharatana and Shah (1983)], the unloading diagrams of fracture specimens do not point to the origin. Rather, a significant residual displacement 03 , approximately as large as seen in Fig. 2, remains. Substituting (9) into (8) and noting that $w_{r} / C_{u}=P$, we get

$$
d \Pi=-\frac{P^{2}}{2} d C_{u}+P d w_{r}-P d w
$$

Now we note that the irreversible (nonrecoverable) deflection is

$$
w_{i r}=w-w_{r}
$$

which is equal to the length of the segment 03 in Fig. 2. Thus, (10) yields

$$
d \Pi=-\frac{P^{2}}{2} d C_{u}-P d w_{i r}
$$

The terms of this equation may be graphically interpreted as

$$
\frac{P^{2}}{2} d C_{u}=d \mathrm{~A}_{3453} ; \quad P d w_{i r}=d A_{35263}
$$

Here $d A_{3453}$ and $d A_{35263}$ represent the areas of the infinitesimal triangle and parallelogram shown in Fig. 2. The sum of all these infinitesimal areas corresponding to all the points on the load-deflection curve is equal to the total area $A$ under the load-deflection curve, i.e.

$$
A=\int_{w=0}^{\infty}\left[d A_{3453}(w)+d A_{35263}(w)\right]
$$

The total energy dissipated by fracturing is obtained as

$$
W_{f}=\int_{w=0}^{\infty} d A_{3453}(w)=A-\int_{w=0}^{\infty} d A_{35263}(w)
$$

Thus $W_{f}$ represents the sum of all the aforementioned elemental triangular areas. Note that the areas of the elemental parallelograms, representing $d A_{35263}$, are excluded. The energy dissipation corresponding to these areas is not caused by the fracturing process. Fracturing (including damage) is the inelastic deformation that changes specimen stiffness, whereas plasticity and frictional slip are the inelastic deformations that do not change specimen stiffness.

As is well-known, the rate of energy release from a fracturing specimen, defined as $G=-\partial \Pi / \partial a$, may be expressed as follows [e.g., Broek (1982, 1986), Kanninen and Popelar (1985), and Bažant and Cedolin (1991), chapter 12]:

$$
G=\frac{P^{2}}{2 b} \frac{d C}{d a}
$$

in which $a=$ crack length (Fig. 1); and $C=$ specimen compliance. When the energy dissipated by plasticity and friction is negligible, $C$ may be taken as the secant compliance. However, when this energy is significant, $C$ must be replaced in this equation by the unloading compliance $C_{u}$. Proof: with $C$ $=C_{u}$, (16) may be written as

$$
-b G d a=-\frac{P^{2}}{2} d C_{u}
$$

The first term in (12) (representing the decrease, due to fracture, of the recoverable part of potential energy) coincides with (17) if and only if $C_{u}$ has been substituted for $C$.

The basic physical characteristic of crack formation in an elastic material is that upon unloading the crack closes and the deformation due to the crack is completely recovered. Cognizant of this fact, let us now define the pure fracture energy
$G_{p f}$ as the energy actually dissipated by the physical process of fracturing. If hypotheses 1 and 2 of the cohesive-crack model are valid and if we further introduce the hypothesis that there exists a unique function $C_{u}(w)$ (hypothesis 3 ), we have

$$
G_{p f}=\frac{1}{b\left(D-a_{0}\right)} \int_{0}^{\infty} \frac{1}{2}[P(w)]^{2} d C_{w}(w)
$$

This value coincides with $G_{f}$ according to (3) with (4) if and only if the unloading diagrams point to the origin. To determine $G_{p s}$, obviously it is necessary to measure the unloading compliance $C_{u}$ at a sufficient number of points on the loaddeflection curve. In practice, $G_{p f}<G_{f}$. According to the unloading slopes reported by Wecharatana and Shah (1983), $G_{p f}$ is between $1 / 4$ and $1 / 2$ of $G_{f}$.

Because the final crack is a line, one may distinguish in the cohesive-crack model plastic deformations within the crack, caused by the crack-bridging cohesive stresses, and plastic deformations in the material near the crack, even though such a distinction is very difficult to make in the real fracture process. The energy dissipated near the cohesive crack due to inelastic behavior of the material cannot be decoupled from the energy dissipated by plasticity in the crack, nor can the energy whose recovery may be prevented by fragments or debris that may have accumulated within the crack. Even though formation of fragments is, in the microscopic physical sense, a fracture process, on the macroscale it plays the same role as plasticity; it increases deformations during monotonic loading and (unless the fragments would be removed by outside intervention, which cannot be described by material laws) it blocks subsequent crack closure.

Planas and Elices (1989) show that the effect of inelastic behavior can be described by the following expression for the apparent fracture energy:

$$
G_{f a}=G_{f 0}\left[1+\psi\left(D / l_{0}\right)\right] ; \quad l_{0}=E^{\prime} G_{f} / f_{f_{2}}^{\prime}
$$

where the fracture energy corresponding to the cohesive crack alone is now relabeled as $G_{f 0} ; l_{0}=$ the characteristic process zone size; $E^{\prime}=$ effective elastic modulus; $f_{t}^{\prime}=$ tensile strength; and $\psi=$ an increasing function depending on the inelastic constitutive law and the specimen geometry. An important point is that the limit $\psi(\infty)$ for infinite specimen size is bounded and does not depend on the specimen geometry, and so is a material constant. Thus the value $G_{f a}^{\infty}=G_{f 0}[1+\psi(\infty)]$ is an effective fracture energy representing the crack growth resistance for a very large structure. By perturbation theory, Planas et al. (1992) estimated for a certain concrete that $\psi(\infty) \approx$ 0.0445 , which is a rather small correction.

\section{IMPROVEMENT MITIGATING EFFECT OF VARIABILITY OF $\Gamma(x)$}

As already mentioned, another problem in determining the fracture energy $G_{f}$ as an intrinsic material property is that, in quasibrittle materials, the fracture process zone (FPZ in Fig. 1) is not a line but has a certain width. The width may vary during fracture propagation. This must cause $\Gamma$ to vary because the opening displacement of the cohesive crack represents the total fracturing deformation accumulated across the width of the fracture process zone, and the wider this zone, the larger the accumulated displacement. The variation of this width obviously must be most pronounced in the initial stage of crack propagation from the notch, as well as in the terminal stage in which the crack approaches the opposite end of the ligament. During the intermediate stage, in which the crack tip is remote from both the notch and the opposite end of the ligament, the variation of $\Gamma$ should be the least. Since the assumption of constant $\Gamma$ is implicit to the work-of-fracture method, the error of this method should be reduced by aver- 

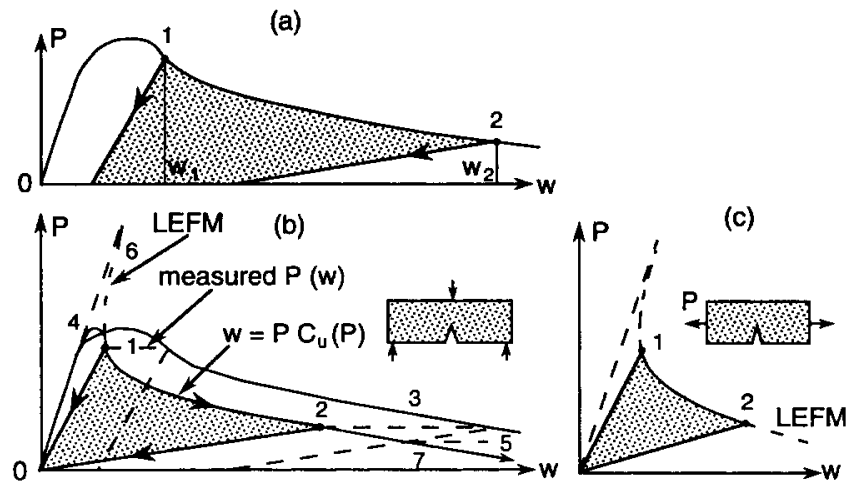

FIG. 3. Partial Matching of LEFM Load-Deflectlon Curve to Measured Curve Adjusted to Elim Inate Plastic-Frictional Energy Dissipation

aging $\Gamma(x)$ over only the intermediate stage, which begins and ends at some crack lengths $a_{1}$ and $a_{2}$, corresponding to points 1 and 2 in Fig. 3 with deflections $w_{1}, w_{2}$ and loads $P_{1}, P_{2}$. Thus it would be more accurate to use the definitions

$$
\begin{aligned}
G_{p f} & =\frac{1}{b\left[a\left(w_{2}\right)-a\left(w_{1}\right)\right]} \int_{w_{1}}^{w_{2}} \frac{1}{2}[P(w)]^{2} d C_{u}(w) \\
& =\frac{1}{b\left[a\left(w_{2}\right)-a\left(w_{1}\right)\right]} \int_{w_{1}}^{w_{2}} d A_{3453}(w)
\end{aligned}
$$

and

$$
\begin{aligned}
G_{f} & =\frac{1}{b\left[a\left(w_{2}\right)-a\left(w_{1}\right)\right]} \int_{w_{1}}^{w_{2}}\left\{\frac{1}{2}[P(w)]^{2} d C_{w}(w)+P w_{i r}\right\} \\
& =\frac{1}{b\left[a\left(w_{2}\right)-a\left(w_{1}\right)\right]} \int_{w_{1}}^{w_{2}}\left[d A_{3453}(w)+d A_{35263}(w)\right]
\end{aligned}
$$

Because (from Fig. 1) $d w_{i r}=d w-d w_{e}-d w_{r}=d w-C_{u} d P$ $-P d C_{u}=d w-(d P / d w) d w-P\left(d C_{u} / d w\right) d w$, the last integral may be transformed by integrating by parts. This provides

$$
\begin{aligned}
G_{f} & =\frac{1}{b\left[a\left(w_{2}\right)-a\left(w_{1}\right)\right]}\left[\frac{1}{2} P_{1}^{2} C_{u}\left(P_{1}\right)\right. \\
& \left.-\frac{1}{2} P_{2}^{2} C_{u}\left(P_{2}\right)+\int_{w_{1}}^{w_{2}} P(w) d w\right] \\
& =\frac{1}{b\left[a\left(w_{2}\right)-a\left(w_{1}\right)\right]} A_{12 m n}
\end{aligned}
$$

where $A_{12 m n}=$ area shaded in Fig. 3, limited by the load-deflection curve and the lines of unloading from points 1 and 2 .

The problem, of course, is how to determine points 1 and 2 and the corresponding $w_{1}$ and $w_{2}$. This can be approximately done by equivalent LEFM. The effective crack length $a$ may be calculated as the LEFM crack length for which the specimen has the same compliance as the measured $C_{u}$. However, for comparisons with LEFM, the plastic-frictional displacements need to be eliminated from the actually measured curve 03 , transforming it to curve 04125 , which involves only deflections due to elasticity and fracture. This may be done graphically, by shifting the points of the actual load-deflection curve leftward, as shown in Fig. 3, so as to make the zero point of unloading diagrams coincide with the origin 0 . This yields the transformed curve 04125 . But better this curve is obtained analytically, according to the equation

$$
w_{e f}(P)=P C_{u}(P)
$$

in which $C_{4}$ is defined as a function of $P$ rather than $w$; and $w_{e f}$ as a function of $P$ represents the transformed curve 04125 .
The LEFM load-deflection curve for a three-point-bend fracture specimen has the shape of the dashed curve 61270 in Fig. 3 [e.g., Bažant (1987a,b) or Bažant and Cedolin (1991, chapter 12)]. This curve has the initial elastic tangent as its asymptote at $P \rightarrow \infty$. The reason that neither the real loaddeflection curve 03 nor the transformed curve 04125 exhibit this asymptotic property is the finiteness of the fracture process zone length. The LEFM curve 6127 may be optimally matched to the transformed curve 04125 , and the segment 12 over which both curves approximately coincide corresponds to the that part of response during which $\Gamma(x)$ is approximately constant (otherwise the LEFM solution, which by definition corresponds to constant $\Gamma$, could not be matched closely). According to the LEFM formula for this matched LEFM curve [e.g., Bažant $(1987 \mathrm{a}, \mathrm{b})$ ], it is then possible to determine the crack lengths $a_{1}$ and $a_{2}$ corresponding to points 1 and 2 , which makes it possible to use the formulas (20) and (21). To do the calculations, the unloading compliancies $C_{k}$ need to be measured for a series of values of $w$, and then the dependence of $C_{u}$ on $w$ needs to be approximated by a suitable simple expression such as a polynomial.

If the specimen is very large, point 1 should lie quite close to the peak load point and curve segment 12 should be relatively long. If the specimen is not large enough, segment 12 may be too short or might not even exist, in which case no matching by the LEFM load-deflection curve is possible. The question now is whether a long enough segment 12 can be obtained for reasonable sizes of laboratory specimens.

If a long enough segment 12 exists, then the complete loaddeflection diagram $P(w)$ is not needed for determining $G_{f}$. This would be a welcome conclusion, because measurement of the long tail of the load-deflection diagram is not easy and its estimation introduces additional error.

\section{COMPARISON TO $J$-INTEGRAL APPROACH TO FRACTURE OF METALS}

In the context of $J$-integral approximation for energy-release rate in small-scale yielding of elastoplastic metals (Rice 1968; Budianski and Rice 1973; Hutchinson 1979), a method to measure the critical energy release by energy difference between two specimens of different notch length $a_{0}$ has been introduced. It is instructive to discuss this ingeneous method in the present context.

Because in absence of plastic strains the complementary energy of the specimen in equilibrium is $\Pi^{*}=P^{2} C / 2$, the energyrelease rate may be expressed as $\partial \Pi * / \partial a=\left(P^{2} / 2\right) \partial C / \partial a$, which yields (16). This may be rewritten as $d \Pi^{*}=\left(P^{2} / 2\right) d C=P d u /$ 2 , where $d u=P d C$. So, in the absence of plastic strain, $d \Pi^{*}$ is the area of the shaded triangle 0120 [Fig. 4(a)] between the
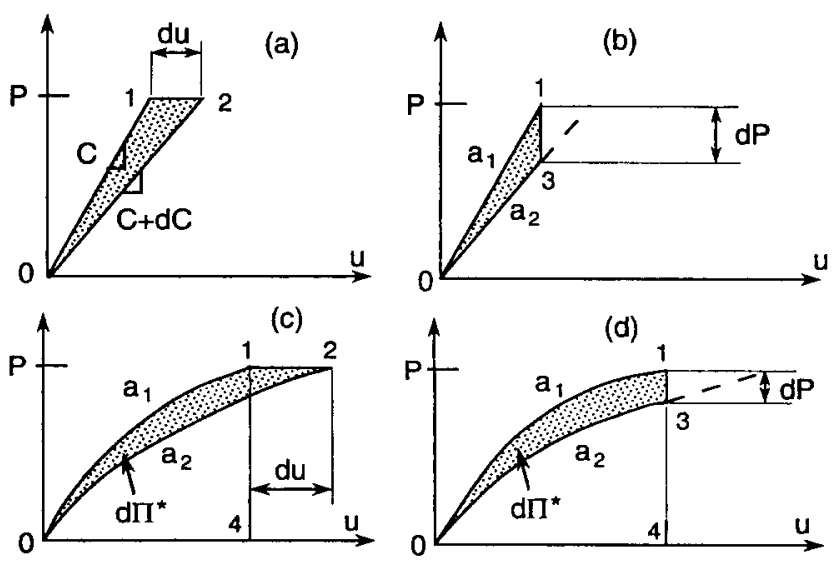

FIG. 4. Linear and Nonlinear Elastic Load-Deflection Curves for Two Specimens with Slightly Different Crack Lengths $a_{1}$ and $a_{2}$ 


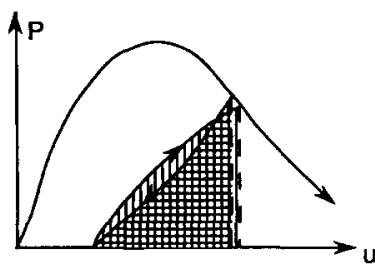

(a)

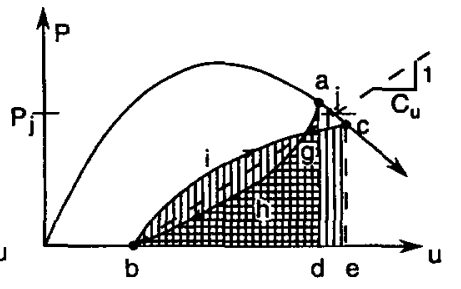

(b)
FIG. 5. Handling of Curvature of Unloading and Reloading DIagrams: (a) Fast Loading; (b) Slow Loading

linear-elastic load-displacement diagrams for crack length $a=$ $a_{1}$ and $a=a_{2}=a+d a$. Since $d a$ is infinitesimal, this area is also equal to the area of the triangle 0130 in Fig. 5(b), which may be written as $u d P / 2=u^{2} d K / 2$, where $K=1 / C=$ specimen stiffness. Because in equilibrium $\Pi=P u / 2=K u^{2} / 2$, the energy release rate also is

$$
G=\left[\frac{\partial \Pi}{\partial a}\right]_{u}=\frac{u^{2}}{2} \frac{d K(a)}{d a}
$$

This means that $d \Pi=\left(u^{2} / 2\right) d K$, which is the area of triangle 0130 in Fig. 4(b).

Eq. (16) and (24) have been generalized to nonlinear elastic behavior, which represents reversible nonlinear deformations but can approximately be assumed for fracture of elastoplastic metals with small-scale yielding. In that case, the potential energy is $\Pi=\int_{0}^{u} P\left(u^{\prime}, a\right) d u^{\prime}=$ area 0140 in Fig. 4(c). In the case of nonlinear elastic behavior, the energy-release rate represents more generally the $J$-integral, which reduces to $G$ in the case of linear elastic behavior. We have

$$
b J=-\left[\frac{\partial \Pi}{\partial a}\right]_{\mu}=-\int_{0}^{\mu} \frac{\partial P\left(u^{\prime}, a\right)}{\partial a} d u
$$

Considering two identical specimens with slightly different crack lengths $a_{1}$ and $a_{2}$, we may use the approximation

$$
b J=\frac{1}{a_{2}-a_{1}}\left[\int_{0}^{u} P\left(u^{\prime}, a_{1}\right) d u^{\prime}-\int_{0}^{u} P\left(u^{\prime}, a_{2}\right) d u^{\prime}\right]
$$

where $a_{1}, a_{2}=$ constants. Thus, $J d a$ (with $d a=a_{2}-a_{1}$ ) represents the shaded area 0120 between the load-deflection curves 01 and 02 for these two specimens (Rice 1968; Budianski and Rice 1973). Alternatively, because for nonlinear elastic behavior $\Pi^{*}=\int_{0}^{P} u\left(P^{\prime}, a\right) d P^{\prime}$

$$
b J=\left[\frac{\partial \Pi^{*}}{\partial a}\right]=\int_{0}^{a} \frac{\partial u\left(P^{\prime}, a\right)}{\partial a} d P^{\prime}
$$

For specimens with slightly different crack lengths, this means that, approximately (Rice 1968; Budianski and Rice 1973)

$$
b J=\frac{1}{a_{2}-a_{1}}\left[\int_{0}^{P} u\left(P^{\prime}, a_{2}\right) d P^{\prime}-\int_{0}^{P} u\left(P^{\prime}, a_{1}\right) d P^{\prime}\right]
$$

which represents the shaded area 0130 between the load-deflection curves of two specimens with crack lengths $a_{1}$ and $a_{2}$, shown in Fig. 4(d). Note that the difference of this area from that in Fig. 4(c) is negligible (higher-order small) for $d a \rightarrow 0$.

The equivalence of (25) and (27) may also be demonstrated by noting that $b J d a=-d \Pi=-(d U-d W)=-\left[\int_{0}^{u} P\left(u^{\prime}, a\right)\right.$ $\left.d u^{\prime}-P u\right]=-\int_{0}^{u}[d(P u)-u d P]+P u=-P u+\int_{0}^{P} u\left(P^{\prime}\right.$, a) $d P^{\prime}+P u=d \Pi^{*}$, in which we integrated by parts; $W=$ work of dead (gravity) load, or $-W=$ potential energy of gravity load.
Eqs. (26) and (27) have been used as the basis of measurement of $J$ in elastoplastic metals under the assumption of small-scale yielding. Note that the energy-release rate determined in this manner includes the energies dissipated by both fracturing and plasticity at the crack tip. These equations have also been applied to concrete. However, such applications are unjustified, for several reasons: (1) The proper value of the effective crack length $a$ is unclear and cannot be kept constant during loading, because (in normal-size fracture specimens) the fracture process zone is too large; (2) $P$ depends not only on $u$ and notch length $a_{0}$, but also on the crack extension $c=$ $a-a_{0} ;$ and (3) these equations are not compatible with the cohesive-crack model.

\section{TIME-DEPENDENT EFFECTS AND REVERSE PLASTICITY}

Ideally, the unloading load-deflection diagram would be straight, as shown by lines 13 or 26 in Fig. 2. In reality, however, the unloading as well as reloading diagrams are curved, as shown in Fig. 5. Although the difference between the start and end points $a$ and $b$ of an unload-reload cycle is partly due to fracture growth, as is well-known from fatigue tests, the observed curvature is mostly due to the viscoelastic (or creep) response of the material and the rate-dependence of the softening stress-displacement law of the crack. When the unloading and reloading are fast, the curvature of the load-deflection diagrams is small [Fig. 5(a)]; and when slow, the curvature is high [Fig. 5(b)]. Since viscoelastic deformation depends mainly on the load level, the curvature of the unloading and reloading diagrams should be nearly the same at each load level. Therefore, the time-dependent irreversible deflections accumulated during the unloading and during the reloading should be nearly the same.

The energy recovered during unloading is given by the area $a h b d a$, and the energy expended during reloading by the area biceb in Fig. 5. The net energy loss over the unload-reload cycle is given by the difference of these two areas. It represents the area of the loop ghbig plus the area dgced (Fig. 5), and can thus be written as

$$
d W_{\text {visc }}=A_{\text {biceb }}-A_{\text {badb }}=A_{\text {bighb }}+A_{\text {geedg }}
$$

This energy must also be excluded from the calculation of the pure fracturing energy $G_{p} f$.

If the description of fracture is time-independent, as usual in practice, the fracture model must be based on the average effective stiffness for the given range of loading rates. In that case, the proper effective unloading compliance to use is the average inverse slope during unloading and reloading, representing the inverse slope of line $j b$ in Fig. 5, i.e.

$$
C_{u}\left(P_{j}\right) \approx \frac{1}{2}\left(\frac{\overline{b d}}{\overline{a d}}+\frac{\overline{b e}}{\overline{c e}}\right)
$$

Evidently, both the unloading and the reloading diagrams need to be measured to determine $C_{u}$ if viscoelastic deformations take place.

If, however, a time-dependent value of $G_{p f}$ is to be used, $C_{u}$ must be determined as a $C_{u}$-value for extremely fast unloading. This value could be obtained by extrapolation from the slopes measured at different rates of unloading.

Some of the curvature of the unloading diagram, however, might be caused by reverse plasticity (akin to the Bauschinger effect in the plastic stress-strain relations). To eliminate contamination by such effects, the initial value of $C_{u}$ at the start of unloading is the proper value to use. This value must be determined only after the viscoelastic effect has been eliminated from the measured unloading diagram. To take this effect and the time-dependent effects into account, $C_{u}$ should be 
taken as the initial inverse unloading slope for extremely fast unloading.

Formation of fragments and debris within the crack would have the opposite effect on the unloading curvature. As the crack closes, more and more fragments may be expected to come into contact with the opposite crack faces and thus produce gradual stiffening, i.e., progressive increase of unloading slope (or locking behavior). Such behavior is not seen in tests. Therefore it is unlikely that crack locking due to possible fragments would play any significant role.

\section{WHICH FRACTURE ENERGY DEFINITION TO USE?}

One might now be tempted to think that the fracture energy value to use in structural analysis is the pure fracture energy $G_{p f}$. But that depends on how the fracture energy value is used, especially whether the plastic-frictional deformations in the fracture process zone are or are not separately taken into account in structural analysis. Usually they are not. Then, of course, the proper value to use is the classical fracture energy $G_{f}$. If, however, a structure is analyzed by a finite-element program involving either a nonlocal plastic-damage model or a fracture model combined with a plastic-damage constitutive model, then the correct value to use is $G_{p f}$.

The fact that the fracture energy to be used in the usual type of fracture analysis of structures must include the plastic-frictional dissipation in the fracture process zone becomes clear by comparison with Dugdale's (1960) model for fracture in plastic materials. This model can be regarded as the limit case of the cohesive-crack model in which the curve $\varphi(w)$ has a horizontal plateau (of unspecified length) ending with a sudden stress drop. As established for metals, in the case of smallscale yielding, for which the plastic zone at the crack front is small compared to the cross-section dimension, the fracture can be approximately described by LEFM, provided that the fracture energy value includes the energy dissipated by plastic yielding near the crack tip. This is also the case in $J$-integral approach, based on the idea of nonlinear elastic material whose nonlinearity approximates the plasticity of metal.

Furthermore, consider the extrapolation to infinite size. The fracture process zone, in which part of the energy is dissipated by plasticity and friction, is exposed along its boundary to the same near-tip LEFM displacement field, regardless of the specimen geometry. Therefore, the stress and deformation fields within the fracture process zone must be the same for any specimen geometry and the same energy must be dissipated. The rest of the specimen behaves elastically and the fracture process zone occupies an infinitely small fraction of the specimen size. Therefore, LEFM must apply exactly, in the case of infinite specimen size, and the fracture energy of course includes the energy dissipated by plasticity and friction.

It also follows that the fracture-energy value obtained by the size-effect method (that is, by matching the extrapolation to infinite size with LEFM) includes the plastic-frictional dissipation, and thus yields $G_{f}$ rather than $G_{p f}$.

The $G_{f}$ values obtained by previous researchers with the work-of-fracture method have been higher than those obtained by the size-effect method-typically more than twice as high. It was suspected that the cause of this difference might be some innate fault of one or the other method. However, the cause appears to be merely a difference in (1) the implied approximations; and (2) the applicability range. Before closing, let us briefly comment on this issue, but solely from the viewpoint of the cohesive-crack model.

The simple formula called the size-effect law is only approximate. If a size range exceeding 1:20 is considered, this formula approaches the LEFM asymptote too rapidly, which tends to diminish the $G_{f}$-value obtained. The slower the approach to the LEFM asymptote, the higher the $G_{f}$-value ob- tained by extrapolation to infinite size. If the size-effect method is based on the generalized size-effect law (Bažant $1987 \mathrm{a}, \mathrm{b})$ with exponent $r<0.5$, it can yield about the same $G_{f}$ as the large-size extrapolation of the work-of-fracture method (however, $r \neq 1$ might be undesirable for other reasons, e.g., certain implications for the asymptotic $R$-curve).

Often the structural analysis is based on a linear (triangular) softening law for the cohesive crack. The simple size-effect law (for which $r=1$ ) yields the approximately correct value of the area under the triangular softening diagram of this slope. For calculating the maximum loads of typical test specimens [whose load-displacement curves have the shape in Fig. 3(a)], only the initial softening slope of the softening law matters (Elices et al. 1992). It would be incorrect to use with such a softening law the total value of $G_{f}$ including the tail area. However, when the complete post-peak softening response of the structure should be calculated, the tail-including $G_{f}$ value must, of course, be considered.

In the usual size range of concrete fracture specimens, the maximum load of a structure depends only on the initial postpeak slope of the softening stress-crack opening law, but not on the tail. But it would be a mistake to think this is always so. When the structure size tends to infinity, eventually a size is reached for which the maximum load depends on the entire softening curve, including its long tail (and thus on the total value of $G_{f}$ ). This is because, when the ratio of the processzone length to the structure size tends to zero, the stress at the crack mouth also tends to zero regardless of the length of the tail. For this asymptotic case, the shape of the load-deflection curve approaches the LEFM curve 06125 in Fig. 3(b), whose peak point 6 is sharply elevated and followed by snapback. The load-deflection curve must have this shape [and not that given by curve 012 in Fig. 3(a)] if the area under the entire softening stress-crack opening curve, including the tail area, should matter for the peak load.

The foregoing issues call for deeper analysis. However, this would be beyond the scope of this paper.

\section{CONCLUSIONS}

1. The fracture energy obtained by the work-of-fracture method (as well as the size-effect method) is an apparent fracture-energy value, a large part of which represents plastic-frictional energy dissipation in and near the fracture process zone at the crack tip. For normal practical applications, this energy must not be excluded from the fracture-energy value.

2. If the unloading compliance is known for sufficiently many points on the softening load-deflection curve, it is possible to determine the pure fracturing energy, defined as the energy that excludes the plastic-frictional energy dissipation. This fracture-energy value is appropriate for sophisticated finite-element analysis in which the material constitutive model and fracture model separately take into account the plastic-frictional energy dissipation.

3. The measured unloading compliancies to be used in the calculation of the pure fracture energy need to be corrected to eliminate the time-dependent effects (material viscoelasticity) and reverse plasticity.

4. The fracture energy obtained by the work-of-fracture method is the average value of the breaking energy across the ligament. The breaking energy, defined as the energy per unit crack area that is required to break the material, varies along the ligament of a notched cross section of a specimen, which is a source of error in the work-of-fracture method. This variation is most pronounced at the beginning of crack growth near the notch, and at the end as the crack approaches the end of the ligament. The error in the work-of-fracture method can 
be reduced by averaging the breaking energy only over a central portion of the ligament, determined such that the load-deflection curve from which the plastic deflections have been eliminated be optimally matched by the LEFM load-deflection curve. At present, however, it is not clear whether the specimen size required for obtaining a good match for a long-enough portion of the loaddeflection curve would not be impracticably large.

5. The fact that the unloading load-deflection slopes of fracture specimens point far from the origin [as shown in Fig. 2, supported by many tests, e.g., Wecharatana and Shah (1983)], implies that a major part of energy dissipation on the microstructural level must be caused by plastic-frictional processes (such as slip or pull-out of grains or fragments) rather than fracture processes.

\section{ACKNOWLEDGMENTS}

Financial support under National Science Foundation (NSF) grant MSS-9114476 to Northwestern University is gratefully acknowledged. Partial support for some related experimental studies that stimulated the present study has been provided by the Center for Advanced CementBased Materials at Northwestern University. Thanks are due to Jaime Planas of the Technical University of Madrid for his very stimulating critical comments.

\section{APPENDIX. REFERENCES}

Barenblatt, G. I. (1959). "The formation of equilibrium cracks during brittle fracture. General ideas and hypothesis, axially symmetric cracks." Prikl. Mat. Mekh., 23(3), 434-444.

Barenblatt, G. I. (1962). "The mathematical theory of equilibrium cracks in brittle fracture." Adv. Appl. Mech., 7, 55-129.

Bažant, Z. P. (1987a). "Fracture energy of heterogeneous material and similitude." Preprints, SEM-RILEM Int. Conf. on Fracture of Concrete and Rock, held in Houston, Texas, June 1987, S. P. Shah and S. E. Swartz, eds., Soc. for Experimental Mech. (SEM), Bethel, Conn., 390-402.

Bažant, Z. P. (1987b). "'Snapback instability at crack ligament tearing and its implication for fracture micromechanics." Cement and Concrete Res., 17, 951-967.

Bažant, Z. P., and Cedolin, L. (1991). Stability of structures: Elastic, inelastic, fracture and damage theories. Oxford University Press, Inc., New York, N.Y.

Bažant, Z. P., Gettu, R., and Kazemi, M. T. (1991). "Identification of nonlinear fracture properties from size-effect tests and structural analysis based on geometry-dependent R-curves." Int. J. Rock Mech. and Min. Sci., 28(1), 43-51.

Bažant, Z. P., and Kazemi, M. T. (1990). "Determination of fracture energy, process zone length and brittleness number from size effect, with application to rock and concrete." Int. J. Fracture, 44, 111-131.

Bažant, Z. P., and Li, Y.-N. (1995). "Stability of cohesive crack model: I. Energy principles." J. Appl. Mech., 62 (Dec.).

Bažant, Z. P., and Pfeiffer, P. A. (1987). "Determination of fracture energy from size effect and brittleness number." ACI Mat. J., 84, 463480.

Bažant, Z. P., Tabbara, M. R., Kazemi, M. T., and Pijaudier-Cabot, G. (1990). "Random particle model for fracture of aggregate or fiber composites." J. Engrg. Mech., ASCE, 116(8), 1686-1705.

Broek, D. (1982). Elementary engineering fracture mechanics, 3rd Ed., Martinus Nijhoff, Leyden, The Netherlands.

Broek, D. (1986). Elementary engineering fracture mechanics, 4th Ed., Martinus-Nijhoff, Dordrecht, The Netherlands.

Budianski, B., and Rice, J. R. (1973). "Conservation laws and energy release rates." J. Appl. Mech., 40, 201-203.

Dugdale, D. S. (1960). "Yielding of steel sheets containing slits." $J$. Mech. and Phys. of Solids, 8, 100-108.

Elices, M., and Planas, J. (1989). "Chapter 3: material models." Fracture mechanics of concrete structures, L. Elfgren, ed., Chapman \& Hall, London, U.K., 16-66.

Elices, M., Guinea, G. V., and Planas, J. (1992). "Measurement of the fracture energy using three-point bend tests: Part 3-Influence of cutting the $P-\delta$ tail." Mat. and Struct., Paris, France, 25, 327-334.

Guinea, G. V., Planas, J., and Elices, M. (1992). "Measurement of the fracture energy using three-point bend tests: Part 1-Influence of experimental procedures." Mat. and Struct., Paris, France, 212-218.

Hillerborg, A. (1983). "Examples of practical results achieved by means of the fictitious crack model." Preprints, Prager Symp. on Mech. of Geomaterials: Rocks, Concretes, Soils, Z. P. Bažant, ed., Northwestern Univ., Evanston, Ill., 611-614.

Hillerborg, A. (1985). "The theoretical basis of method to determine the fracture energy $G_{f}$ of concrete." Mat. and Struct., Paris, France, $18(106), 291-296$.

Hillerborg, A., Modéer, M., and Petersson, P. E. (1976). "Analysis of crack formation and crack growth in concrete by means of fracture mechanics and finite elements." Cement and Concrete Res., 6, 773782.

Hu, X. Z., and Wittmann, F. H. (1991). "An analytical method to determine the bridging stress transferred within the fracture process zone: I. General theory." Cement and Concrete Res., 21, 1118-1128.

Hu, X. Z., and Wittmann, F. H. (1992). "An analytical method to determine the bridging stress transferred within the fracture process zone: I. Application to mortar." Cement and Concrete Res., 21, 559-570.

Hutchinson, J. W. (1979). A course on nonlinear fracture mechanics. Dept. of Solid Mech., Tech. Univ. of Denmark, Lyngby, Denmark.

Jirásek, M., and Bažant, Z. P. (1994). "Localization analysis of nonlocal model based on crack interactions." J. Engrg. Mech., ASCE, 120(3), $1521-1542$.

Jirásek, M., and Bažant, Z. P. (1995). "Macroscopic fracture characteristics of random particle systems." Int. J. Fracture, 69, 201-228.

Kanninen, M. F., and Popelar, C. H. (1985). Advanced fracture mechanics, Oxford University Press, Inc., New York, N.Y.

Mihashi, H., Nomura, N., Izumi, M., and Wittmann, F. H. (1991). "Size dependence of fracture energy of concrete." Fracture processes in concrete, rocks and ceramics, (Proc., Int. RILEM Conf., Noordwijk, Netherlands), van Mier, Rots, and Bakker, eds., E\&FN Spon, London, U.K., $441-450$.

Nakayama, J. (1965). "Direct measurement of fracture energies of brittle heterogeneous material." J. Am. Ceram. Soc., 48(11).

Petersson, P. E. (1981). "Crack growth and development of fracture zones in plain concrete and similar materials." Rep., TVBM-1006, Div. of Build. Mat.; Lund Inst. of Tech., Lund, Sweden.

Planas, J., and Elices, M. (1988). "Size-effect in concrete structures: Mathematical approximation and experimental validation." Cracking and Damage, Strain Localization and Size Effect: Proc., France-U.S. Workshop, Cachan, France, J. Mazars and Z. P. Bažant, eds., Elsevier, London, U.K., 462-476.

Planas, J., and Elices, M. (1989). "Conceptual and experimental problems in the determination of the fracture energy of concrete." Fracture Toughness and Fracture Energy: Proc., RILEM Int. Workshop, Mihashi et al,, eds., A. A. Balkema, Rotterdam, The Netherlands, 165-181.

Planas, J., Elices, M., and Guinea, G. V. (1992). "Measurement of the fracture energy using three-point bend tests: Part 2-Influence of bulk energy dissipation."' Mat. and Struct., Paris, France, 305-312.

Rice, J. R. (1968). "Mathematical analysis in the mechanics of fracture." Fracture-An advanced treatise, Vol. 2, H. Liebowitz, ed., Academic Press, New York, N.Y., 191-308.

RILEM. (1985). "Determination of the fracture energy of mortar and concrete by means of three-point bend tests on notched beams: TC-50FMC, fracture mechanics of concrete." RILEM Recommendation, Mat. and Struct., Paris, France, 18(106).

Tattersall, H. G., and Tappin, G. (1966). "The work of fracture and its measurements in metals, ceramics and other materials." J. Mat. Sci., 1(3), 296-301.

Wecharatana, M., and Shah, S. P. (1983). "Nonlinear fracture mechanics parameters." Fracture mechanics of concrete, F. H. Wittmann, ed., Elsevier, Amsterdam, The Netherlands, 483-480.

Zubelewicz, A., and Bažant, Z. P. (1987). "Interface modeling of fracture in aggregate composites." J. Engrg. Mech., ASCE, 113(11), 16191630 . 